\title{
SEPARABLE MENGER-REGULAR HAUSDORFF CURVES ARE METRIZABLE
}

\section{J. L. CORNETTE}

ABSTRACT. It is shown that every connected, separable, locally compact and locally peripherally finite Hausdorff space is metrizable.

1. Introduction. A Menger-regular Hausdorff space is a connected Hausdorff topological space with a base for the topology consisting of neighborhoods with finite boundaries. We prove the following:

Theorem 1. If $M$ is a Menger-regular Hausdorff continuum and $A$ is a closed subset of $M$, and $S$ is a dense subset of $M$, then there is a subset $T$ of $A$ which is dense in $A$ such that the cardinality of $T$ is less than or equal to the cardinality of $S$.

Corollary 1.1. If $M$ is a separable Menger-regular Hausdorff continuum, every arc contained in $M$ is metrizable.

Theorem 2. Every separable Menger-regular Hausdorff continuum is metrizable.

Corollary 2.1. Every separable locally compact Menger-regular Hausdorff space is metrizable.

Suppose $M$ is a Menger-regular Hausdorff continuum. Using techniques directly analogous to those for metric continua, it can be shown that each two closed disjoint subsets of $M$ are separated in $M$ by a finite subset of $M$ and that $M$ is locally connected [1, p. 96], [2, p. 107]. Obviously each subcontinuum of $M$ is a Menger-regular Hausdorff continuum and therefore is locally connected. If $O$ is a connected open subset of $M$, and $P$ and $Q$ belong to $O$, there is in $O$ an irreducible continuum $K$ from $P$ to $Q$. Since $K$ is locally connected, each point of $K$ distinct from $P$ and $Q$ is a cutpoint

Received by the editors July 20, 1973.

AMS (MOS) subject classifications (1970). Primary 54D05, 54E35; Secondary $54 \mathrm{~A} 25$.

Key words and phrases. Menger-regular, metrizable, locally connected.

Copyright $\odot$ 1974, American Mathematical Society 
of $K$ and $K$ is therefore a Hausdorff arc. Consequently $M$ is Hausdorff arcwise connected and locally Hausdorff arcwise connected. If $P$ is a point of $M$ and $C$ is a closed subset of $M$, an arc from $P$ to $C$ in $M$ is $\{P\}$ if $P$ belongs to $C$ and if $P$ does not belong to $C$, it is a Hausdorff arc with $P$ as one endpoint, its other endpoint in $C$ and which contains no other point of $C$. We let $N$ denote the positive integers.

2. Proof of Theorem 1. Suppose the hypothesis. Let $\omega$ denote the family of all $W$ such that $W$ is a disjoint collection each member of which is an arc from a point of $S$ to $A$. Then $\omega$ is partially ordered by containment, the union of any chain of elements of $\omega$ is a member of $\omega$ which is an upper bound of that chain, and therefore there is a maximal element $W_{\max }$ of $\omega$.

Let $T=A \cap\left[\bigcup W_{\max }\right]$. The cardinality of $T$ is obviously less than or equal to the cardinality of $S$. We will show that $T$ is dense in $A$.

Suppose $T$ is not dense in $A$ and that $P$ is a point of $A-\operatorname{Cl}(T)$. There is a finite set $F$ separating $P$ from $\mathrm{Cl}(T)$ in $M$, and because the members of $W_{\max }$ are disjoint, $F$ intersects at most a finite number of them. Consequently, $P$ is not a limit point of $\bigcup W_{\max }$ and there exists a connected open set $O$ that contains $P$ and misses $\bigcup W_{\max }$. Then $O$ contains a point $Q$ of $S$ and also contains an arc $\alpha$ from $Q$ to $A$. But $W_{\max } \cup\{\alpha\}$ is a member of $\omega$ which properly contains $W_{\max }$ and this is a contradiction.

Proof of Corollary 1.1. If $\alpha$ is an arc in $M$ and $M$ is separable, then, from Theorem 1, $a$ is separable, and it is known that every separable Hausdorff arc is metrizable [2, p. 31$]$.

3. Proof of Theorem 2. Suppose $M$ is a separable Menger-regular Hausdorff continuum and $\left\{P_{0}, P_{1}, P_{2}, \cdots\right\}$ is a countable sequence of distinct points which is dense in $M$. We define inductively the sequence $\left\{A_{0}, A_{1}\right.$, $\left.A_{2}, \cdots\right\}$.

(i) Let $A_{0}$ denote $\left\{P_{0}\right\}$.

(ii) Suppose $n$ is a nonnegative integer and $A_{i}$ has been defined for $0 \leq i \leq n$. Let $A_{n+1}$ denote an arc from $P_{n+1}$ to $\bigcup^{i}\left\{A_{i} \mid i=0,1, \cdots, n\right\}$. For each $n \geq 0$, let $D_{n}$ denote $\bigcup\left\{A_{i} \mid i=0,1, \cdots, n\right\}$, and let $Q_{n+1}$ denote the point of $A_{n+1}$ that belongs to $D_{n}$. Then for $n \in N, D_{n}$ is the union of a finite number of metric arcs and is itself a metric space, in fact, a metric dendron (locally connected metric continuum which contains no simple closed curve). For $n \geq 0$, define $\rho_{n+1}: D_{n+1} \rightarrow D_{n}$ by

$$
\begin{aligned}
\rho_{n+1}(x) & =x & & \text { if } x \in D_{n}, \\
& =Q_{n+1} & & \text { if } x \in A_{n+1} .
\end{aligned}
$$


It is apparent that, for $n \in N, \rho_{n}$ is continuous, and we let $D_{\infty}$ denote the inverse limit space of the inverse limit system $\left\{D_{n}, \rho_{n}, n \in N\right\}$. Since, for $n \in N, D_{n}$ is a compact metric continuum, $D_{\infty}$ is a compact metric continuum. It can be shown that $D_{\infty}$ is a dendron, but we do not require this.

Suppose $\left(x_{1}, x_{2}, \cdots\right)$ belongs to $D_{\infty}$. For $n \in N$, either $x_{n+1}$ belongs to $D_{n}$, in which case $x_{n+1}=x_{n}$, or $x_{n+1}$ does not belong to $D_{n}$, in which case $x_{n+1}$ belongs to $A_{n+1}$ and $x_{n}$ is $Q_{n+1}$. In the first case we let [ $x_{n}$, $\left.x_{n+1}\right]$ denote $\left\{x_{n}\right\}$, and in the second case we let $\left[x_{n}, x_{n+1}\right]$ denote the subarc of $A_{n+1}$ with endpoints $x_{n}$ and $x_{n+1}$. For $m, n \in N, m<n$, we let $\left[x_{m}\right.$, $\left.x_{n}\right]$ denote $\bigcup\left\{\left[x_{k}, x_{k+1}\right] \mid k=m, n-1\right\}$. By induction arguments which we omit, it can be shown that if $i, j, k \in N, i<j<k$, then

(a) $\left[x_{i}, x_{j}\right]$ is either $\left\{x_{i}\right\}$ or an arc with endpoints $x_{i}$ and $x_{j}$.

(b) $x_{i}$ is the only point of $\left[x_{i}, x_{j}\right]$ which belongs to $D_{i}$.

(c) $x_{j}$ is the only point common to $\left[x_{i}, x_{j}\right]$ and $\left[x_{j}, x_{k}\right]$.

Lemma 1. If $\left(x_{1}, x_{2}, \cdots\right)$ is a point of $D_{\infty}$, there is a point $P$ of $M$ such that $\lim \left[x_{n}, x_{n+1}\right]=\{P\}$.

Proof. The sequence $x_{1}, x_{2}, \cdots$ has at least one cluster point, $P$. Suppose $q_{1}, q_{2}, \cdots$ is a sequence such that, for $n \in N, q_{n} \in\left[x_{n}, x_{n+1}\right]$, and $q_{1}, q_{2}, \cdots$ has a cluster point $Q$ distinct from $P$. There is a finite subset $F$ of $M$ such that $M-F=U \cup V, U$ and $V$ disjoint open subsets of $M$ containing $P$ and $Q$ respectively. There is an infinite increasing sequence $n(1)$, $n(2), \cdots$ such that for $k \in N, x_{n(2 k-1)}$ belongs to $U$ and $q_{n(2 k)}$ belongs to $V$. Then for $k \in N, F$ contains a cutpoint of $\left[x_{n(2 k-1)}, x_{n(2 k+1)}\right]$, and because $F$ is finite, there is a point $f$ of $F$ and there are two integers $i$ and $j$ such that $f$ is a cutpoint of both $\left[x_{n(2 i-1)}, x_{n(2 i+1)}\right]$ and $\left[x_{n(2 j-1)}\right.$, $\left.x_{n(2 j+1)}\right]$. This and statement (c) above lead to a contradiction. It follows that $\{P\}$ is $\lim \left[x_{n}, x_{n+1}\right]$.

We define $\phi: D_{\infty} \rightarrow M$ by: If $x=\left(x_{1}, x_{2}, \cdots\right)$ belongs to $D_{\infty}, \phi(x)=$ $\lim x_{n}$.

It follows from Lemma 1 that $\phi$ is a function. We show that

Lemma 2. $\phi$ is continuous.

Proof. Suppose $x \in D_{\infty}$ and $O$ is an open set in $M$ containing $\phi(x)$. There is an open set $R$ in $M$ which contains $\phi(x)$ such that $\mathrm{Cl}(R)$ is a subset of $O$ and $R$ has a finite boundary $F$. Since $F$ is finite, there is $i \in N$ such that $D_{i}$ contains $F \cap\left[\bigcup\left\{D_{n} \mid n \in N\right\}\right]$. There is an integer $j$ greater than $i$ such that $x_{j}$ belongs to $R$ and there is a connected open subset $U_{j}$ of $D_{j}$ that contains $x_{j}$ and is a subset of $R$. Let $U$ denote the 
points $y$ of $D_{\infty}$ such that the $j$ th coordinate of $y$ belongs to $U_{j}$. Then $U$ is an open subset of $D_{\infty}$ which contains $y$ and we next show that $\phi(U) \subset$ $\mathrm{Cl}(R) \subset O$.

Suppose $y=\left(y_{1}, y_{2}, \cdots\right)$ is a point of $U$ and $\phi(y)$ does not belong to $\mathrm{Cl}(R)$. Then $y_{j}$ belongs to $U_{j}$ and therefore belongs to both $D_{j}$ and $R$. But since $\phi(y)=\lim y_{n}$ does not belong to $\mathrm{Cl}(R)$, there is an integer $k>j$ such that $y_{k}$ does not belong to $\mathrm{Cl}(R)$. But then $F$ must contain a point $y^{\prime}$ of $\left[y_{j}, y_{k}\right] \subset D_{k}$, and since $F \cap D_{k} \subset D_{i} \subset D_{j}, y^{\prime}$ belongs to $D_{j}$. But $y_{j}$ and $y^{\prime}$ are two points of $\left[y_{j}, y_{k}\right]$ that belong to $D_{j}$ and this is a contradiction to (b) above.

Lemma 3. $\phi$ maps $D_{\infty}$ onto $M$.

For $n \in N, \phi$ maps the point $\left(\rho_{1} \cdots \rho_{n-1}\left(P_{n}\right), \rho_{2} \cdots \rho_{n-1}\left(P_{n}\right), \cdots\right.$, $\left.\rho_{n-1}\left(P_{n}\right), P_{n}, P_{n}, \cdots\right)$ onto $P_{n}$. Therefore $\phi\left(D_{\infty}\right)$ is dense in $M$ and since $D_{\infty}$ is compact, $\phi\left(D_{\infty}\right)$ is compact and is therefore $M$.

We have shown that $M$ is the continuous image of a compact metric space, and since $M$ is Hausdorff, it follows that $M$ is metrizable.

Proof of Corollary 2.1. Suppose $M$ is a separable locally compact Menger-regular Hausdorff space. The one point compactification of $M$ is a separable Menger -regular Hausdorff continuum, which by Theorem 2 is metrizable, and it follows that $M$ is metrizable.

\section{REFERENCES}

1. G.T. Whyburn, Analytic topology, Amer. Math. Soc. Colloq. Publ., vol. 28, Amer. Math. Soc., Providence, R.I., 1942. MR 4, 86.

2. R.L. Wilder, Topology of manifolds, Amer. Math. Soc. Colloq. Publ., vol. 32, Amer. Math. Soc., Providence, R.I., 1949. MR 10, 614.

DEPARTMENT OF MATHEMATICS, UNIVERSITI KEBANGSAAN MALAYSIA, KUALA LUMPUR, MALAYSIA

DEPARTMENT OF MATHEMATICS, IOWA STATE UNIVERSITY, AMES, IOWA 50010 (Current address) 